\title{
Local barrier dysfunction identified by confocal laser endomicroscopy predicts relapse in inflammatory bowel disease
}

\author{
R Kiesslich, ${ }^{1}$ C A Duckworth, ${ }^{2}$ D Moussata, ${ }^{3}$ A Gloeckner, ${ }^{1}$ L G Lim, ${ }^{4}$ M Goetz, \\ D M Pritchard, ${ }^{2}$ P R Galle, ${ }^{1}$ M F Neurath, ${ }^{5}$ A J M Watson ${ }^{6}$
}

\begin{abstract}
- Additional materials are published online only. To view these files please visit the journal online (http://gut.bmj. com/content/61/8.toc)
\end{abstract}

${ }^{1}$ Medical Department, Johannes Gutenberg University of Mainz, Mainz, Germany

${ }^{2}$ Department of

Gastroenterology, Institute of

Translational Medicine,

University of Liverpool, UK

${ }^{3}$ Department of

Gastroenterology, Lyon Sud Hospital, Claude Bernard

University, Pierre Benite, France

${ }^{4}$ Department of

Gastroenterology and

Hepatology, National University

Health System, Yong Yoo Lin

School of Medicine, National

University of Singapore,

Singapore

${ }^{5}$ Department of Medicine I,

University of

Erlangen-Nuremberg, Erlangen, Germany

${ }^{6}$ Faculty of Health, Norwich Medical School, University of East Anglia, Norwich Research Park, Norwich UK

\section{Correspondence to}

Professor A J M Watson, Eliz

Fry Bldg. 2.14, Norwich Medical

School, University of East

Anglia, Norwich NR4 7TJ, UK;

alastair.watson@uea.ac.uk

RK and CAD share first authorship.

Parts of the study were presented at Digestive Disease Week 2009 at the plenary session and at Digestive

Disease Week 2011.

Revised 9 October 2011 Accepted 10 October 2011 Published Online First 24 November 2011

\section{UNLOCKID}

This paper is freely available online under the BMJ Journals unlocked scheme, see http:// gut.bmj.com/site/about/ unlocked.xhtml

\section{ABSTRACT}

Objectives Loss of intestinal barrier function plays an important role in the pathogenesis of inflammatory bowel disease (IBD). Shedding of intestinal epithelial cells is a potential cause of barrier loss during inflammation. The objectives of the study were (1) to determine whether cell shedding and barrier loss in humans can be detected by confocal endomicroscopy and (2) whether these parameters predict relapse of IBD.

Methods Confocal endomicroscopy was performed in IBD and control patients using intravenous fluorescein to determine the relationship between cell shedding and local barrier dysfunction. A grading system based on appearances at confocal endomicroscopy in humans was devised and used to predict relapse in a prospective pilot study of 47 patients with ulcerative colitis and 11 patients with Crohn's disease.

Results Confocal endomicroscopy in humans detected shedding epithelial cells and local barrier defects as plumes of fluorescein effluxing through the epithelium. Mouse experiments demonstrated inward flow through some leakage-associated shedding events, which was increased when luminal osmolarity was decreased. In IBD patients in clinical remission, increased cell shedding with fluorescein leakage was associated with subsequent relapse within 12 months after endomicroscopic examination $(p<0.001)$. The sensitivity, specificity and accuracy for the grading system to predict a flare were $62.5 \%$ (95\% Cl $40.8 \%$ to $80.4 \%), 91.2 \%$ (95\% Cl 75.2 to 97.7$)$ and $79 \%(95 \% \mathrm{Cl} 57.7$ to 95.5$)$, respectively.

Conclusions Cell shedding and barrier loss detected by confocal endomicroscopy predicts relapse of IBD and has potential as a diagnostic tool for the management of the disease.

\section{INTRODUCTION}

Defects in intestinal barrier function have been associated with intestinal inflammatory disease. ${ }^{1-4}$ In Crohn's disease (CD) increased intestinal permeability has been observed in patients and their relatives. ${ }^{5-7}$ Increased intestinal permeability is also a prognostic indicator of relapse. ${ }^{8}$ Further data implicating barrier defects in disease pathogenesis is altered expression of tight junction proteins in patients with CD. ${ }^{9}$ Moreover, inhibition of caveolar endocytosis of occludin out of the tight junction reduces the tumour necrosis factor (TNF) induced barrier loss and water secretion. ${ }^{10}$

\section{Significance of this study}

What is already known on this subject?

- Loss of intestinal barrier function plays an important role in the pathogenesis of inflammatory bowel disease (IBD).

- Intestinal epithelial cells are constantly shed leaving gaps in the epithelial monolayer.

- In the uninflamed intestine these gaps are sealed by a redistribution of tight junction proteins.

- In the inflamed intestine this sealing mechanism starts to fail with loss of barrier function.

What are the new findings?

- Loss of intestinal barrier function at sites of cell shedding can be detected by confocal endomicroscopy with intravenous fluorescein.

- The direction of flow through defects in intestinal epithelium is influenced by the osmolarity of the intestinal luminal contents.

- A simple grading system was devised to summarise the severity of barrier loss and cell shedding at confocal endomicroscopy in humans.

- This grading system predicts relapse of IBD over a 12-month period.

\section{How might it impact on clinical practice in the} foreseeable future?

- Assessment of barrier function and cell shedding by confocal endomicroscopy promises to be a simple, fast and practical method of predicting relapse of IBD.

Crucially, primary changes of tight junction permeability correlate with the severity of experimental colitis. ${ }^{11} 12$ Thus an intact intestinal barrier separating the host from the intestinal contents is essential for gut health.

The intestinal barrier comprises epithelial cells and the tight junctions between them. The epithelial cells migrate from stem cells at the base of crypts to the small intestinal villi or the surface of the colon from where they are shed. Our studies in mice have shown that epithelial cell shedding leaves a gap or discontinuity in the epithelium that resolves in most cases in $8-12 \mathrm{~min}$, potentially 
challenging the epithelial barrier. ${ }^{13}$ These gaps can be detected in humans by confocal laser endomicroscopy (CLE) using the DNA fluorescence dye acriflavine applied to the mucosal surface of the intestine. ${ }^{14}$ These observations in mice and humans raise the fundamental biological question of how barrier function is maintained during epithelial shedding. ${ }^{15}$ We have recently shown that during cell shedding there is a redistribution of component proteins of the tight junction, including $\mathrm{ZO}-1$ and occludin, to surround the basolateral surface of the shedding cell in the form of a funnel, thereby preserving the barrier at the shedding site. ${ }^{13} 16$ We have also shown that cell shedding is increased by high concentrations of TNF and that in this stressed situation the intestinal barrier sometimes fails. ${ }^{14}$ These observations suggest that cell shedding might be increased in inflammatory bowel disease (IBD), as has been recently shown. ${ }^{17}$

A number of methods have been developed for the noninvasive measurement of permeability of the paracellular pathway of absorption and secretion. ${ }^{18}$ These are based on the absorption of small molecular weight saccharides or chromiumlabelled EDTA and measurement of their appearance in the urine. They have proved useful in providing an integrated measurement of barrier function along a long segment of gut. For example, sucrose absorption measures gastroduodenal permeability $^{19}$ whereas lactulose/mannitol gives a measure of small intestinal permeability. ${ }^{20}$ These techniques have been successful in the examination of permeability changes in large populations of patients with IBD. ${ }^{121}$

More detailed analysis of intestinal epithelial permeability can be achieved using in vitro techniques on human biopsies including measurement of transepithelial electrical resistance and the flux of ${ }^{3} \mathrm{H}$-mannitol. ${ }^{22}{ }^{23}$ Spatial information on the location of barrier defects can be obtained with more sophisticated electrical techniques including conductance scanning and impedance spectroscopy. ${ }^{24}$ While these techniques have proved useful, they do not provide optical information on the sites of barrier dysfunction. ${ }^{25}$ Direct visualisation of barrier defects is possible with bench top confocal microscopy, which has been used with success to image the influx of fluorescent probes and bacteria from the intestinal lumen. ${ }^{26} 27$

The aims of this study were, first, to determine whether cell shedding and loss of barrier function in humans can be visualised by confocal endomicroscopy and, second, whether these parameters predict relapse of IBD. We find that CLE can detect cell shedding and barrier loss. Furthermore a prospective pilot study suggests that loss of barrier function detected by CLE predicts subsequent relapse of IBD.

\section{MATERIAL AND METHODS \\ Retrospective human study: cell shedding, gap formation and barrier loss in humans}

In a retrospective study of archived CLE images of the terminal ileum ( $\geq 30$ endomicroscopic images per patient; including surface epithelial cell layer) 13 patients with IBD in clinical remission ( $C D n=7$, ulcerative colitis (UC) $n=6$ ) and 12 control patients (colon cancer screening) were examined (online supplementary table 1). Only endomicroscopic images of the terminal ileum were selected for analysis of local barrier dysfunction because single epithelial cell layer can be observed in vertical and horizontal axis and only minimal overlay of cellular structures occur. Endomicroscopic images were selected based on the presence of epithelial cell layer and intestinal lumen, and absence of movement artefacts.
The diagnosis of CD or UC was made on clinical, endoscopic and histological criteria. There was no significant difference in age or gender between the patient groups (online supplementary table 1). For the patients with $\mathrm{CD}$, remission was defined by a CD activity index (CDAI) $\leq 150$ and UC by a Clinical Activity Index (CAI) $\leq 1 . .^{28} 29$ The control group comprised patients without any intestinal symptoms (despite possible occult bleeding confirmed by at least one positive faecal occult blood test) undergoing endoscopic examination for colorectal cancer screening. Patients with acute gastrointestinal bleeding, coagulopathy (pro-thrombin time $<50 \%$ control; partial thromboplastin time $>50 \mathrm{~s}$ ), impaired renal function (creatinine $>1.2 \mathrm{mg} / \mathrm{dl}$ ), pregnancy or breast feeding, known allergy to fluorescein or inability to give consent were excluded.

\section{Prospective pilot study of the predictive value of cell shedding} gap formation and barrier loss for relapse of IBD

Patients in clinical remission (CDAI $\leq 150$ and a CAI $\leq 1)$ with surveillance colonoscopy based on chronic inflammation (CD or UC) within the colon for more than 8 years were offered to undergo endomicroscopic examination (recruitment period January 2007 to September 2009). Patients were consecutively included for analysis if the terminal ileum could be endomicroscopically imaged. Inclusion criteria were presence of IBD for $>8$ years, absence of macroscopic visible inflammatory changes in the colon or terminal ileum. Exclusion criteria comprised active acute gastrointestinal bleeding, coagulopathy (prothrombin time $<50 \%$ control; partial thromboplastin time $>50 \mathrm{~s}$ ), impaired renal function (creatinine $>1.2 \mathrm{mg} / \mathrm{dl}$ ), pregnancy or breast feeding, known allergy to fluorescein or inability to give consent.

One hundred and thirty-five patients were screened. The majority could not be included because of visible inflammatory changes (61) or impossibility to reach the terminal ileum (16). Fifty-eight patients with IBD $(C D=11, U C n=47)$ in clinical and mucosal remission were finally analysed (online supplementary table 2). The Watson grade was determined by examination of the stored images collected of the terminal ileum at CLE by two blinded investigators. The patients were prospectively followed (monthly follow-up) for 12 months using the CDAI in CD patients and the CAI in UC patients. A flare was defined as a $\mathrm{CDAI}>150$ and a $\mathrm{CAI}>3$. The identified patients and their primary care physicians were invited to participate in the human studies and informed consent was obtained.

The local ethical committee in Rheinland-Pfalz, Germany, approved (No. 837.364.07) both the retrospective and prospective human studies.

\section{Method of CLE and imaging of local barrier dysfunction}

For all the human examinations, the Pentax EC3870K endomicroscope colonoscope (Pentax, Tokyo) was used, enabling in vivo surface and subsurface (z-axis) imaging at (sub)cellular resolution imaging during ongoing video endoscopy. A single intravenous bolus of $5 \mathrm{ml} 10 \%$ sodium fluorescein $(10 \%-$ Novartis Pharma, Germany), was used as a systemic contrast agent and permeability probe. For all the patients, a conscious sedation using propofol was administered for colonoscopy.

Physiological and pathophysiological changes within the lumen and the mucosa of the terminal ileum were inspected for up to $8 \mathrm{~min}$ after injection of fluorescein. Images stacks (Z-stacks) at a given area were collected from the surface of the epithelium down to the lamina propria (optical biopsy). The endomicroscopic images were digitally stored and were analysed 
after the procedure. Images were initially analysed after the examination using Pentax software after the procedure.

\section{Endomicroscopic image analysis}

For the retrospective study all suitable endomicroscopic images of the terminal ileum were analysed for the presence of gaps, microerosions and fluorescein leakage. The overall perimeter of the villi in each image was calculated using Image J (imagej. nih.gov.il/) and the amount of affected cells (per perimeter) was defined (in percentage per optical analysis, which represents a z-stack of images in a given mucosal area). This detailed analysis was used to devise the Watson grading system (see below).

\section{Description and validation grading of cell shedding and barrier loss from endomicroscopic images}

An endomicroscopic grading system (Watson grade) was devised to summarise the degree of local barrier dysfunction in IBD based on the amount of cell shedding and the intensity of the luminal fluorescein signal on single good quality images from four separated field of view of the terminal ileum (table 1). Only areas that were macroscopically normal on the standard colonoscopy were selected for endomicroscopic imaging. Great care was taken to avoid taking images from areas of mucosa with suction artefacts or endoscopic trauma. Images were graded according to table 1 . The severity of barrier loss and microerosions was discrepant between the images or between the two blinded investigators the grade was calculated on the most severe changes.

\section{Confocal laser microscopy of mice}

Confocal laser microscopy of mice was performed as previously described. $^{26}$ Fluorescein isothiocyanate (FITC) conjugated dextran (4 kDa; $20 \mu \mathrm{g} / \mathrm{ml}$; Sigma) was used as a luminal dye to detect inward flow. Intravenous Alexa-fluor conjugated dextran 647 (10 kDa; 4 mg/kg; Invitrogen, Paisley, Scotland) was used to detect outward flow. Hoechst 33342 ( $5 \mathrm{mg} / \mathrm{kg}$; Invitrogen) was used as a nuclear dye. See the online supplemental methods file for further details.

\section{Statistics}

Descriptive data are presented as means \pm SEM. Comparisons between the IBD group and normal control patients were made using $t$ tests and ANOVA tests. Comparison of the percentage of patients in remission on the basis of their Watson grade was

Table 1 Endomicroscopic grade (Watson grade) for in vivo identification of local barrier dysfunction

\begin{tabular}{lll}
\hline & Cell shedding & Local barrier dysfunction \\
\hline I. Normal & $\begin{array}{l}\text { Cell shedding confined to } \\
\text { single cells per shedding } \\
\text { site (eg, figure 1C or D) }\end{array}$ & None \\
$\begin{array}{l}\text { II. Functional } \\
\text { defect }\end{array}$ & $\begin{array}{l}\text { Cell shedding confined to } \\
\text { single cells per shedding } \\
\text { site }\end{array}$ & $\begin{array}{l}\text { Fluorescein signal visible in the } \\
\text { intestinal lumen with an intensity } \\
\text { the same or brighter than the } \\
\text { epithelium or fluorescein plumes } \\
\text { out of the epithelium into the lumen } \\
\text { (eg, figure 2D) }\end{array}$ \\
$\begin{array}{ll}\text { III. Structural } \\
\text { defect }\end{array}$ & $\begin{array}{l}\text { Microerosions in any field. } \\
\text { Microerosion is defined when } \\
\text { the lamina propria is exposed } \\
\text { to the lumen with multiple } \\
\text { cells being shed per site } \\
\text { (eg, figure 2E) }\end{array}$ & $\begin{array}{l}\text { Fluorescein signal visible in the } \\
\text { intestinal lumen with an intensity } \\
\text { the same or brighter than the } \\
\text { epithelium or fluorescein plumes } \\
\text { out of the epithelium into the } \\
\text { lumen (eg, figure 2E) }\end{array}$ \\
\hline
\end{tabular}

See Material and methods. made using the Kaplan-Meier method and analysed by LogRank test. Significance level $\alpha$ was set to $5 \%$. Statistics were computed using the statistical analysis system SAS (release 6.08, SAS Institute Inc.).

\section{RESULTS \\ Cell shedding can be detected by CLE using fluorescein as contrast agent}

We have shown previously that CLE can identify cell shedding events using acriflavine applied topically to the mucosal surface. ${ }^{14}$ We now demonstrate that cell shedding can also be identified using intravenous fluorescein as the contrast agent. Intravenous fluorescein enables bright fluorescent imaging of capillary arcades within the villus (figure 1A). Prior to shedding, epithelial cells become permeable to fluorescein (figure 1B) and become intensely fluorescent as shedding proceeds (figure $1 \mathrm{C}-\mathrm{E})$. Fluorescent shedding cells can be readily identified when viewed en face during confocal endomicroscopy (figure 1F).

\section{Loss of local barrier function}

In the healthy intestine although fluorescein enters the lateral intercellular spaces up to the apical border, there is no escape into the lumen, presumably because of constraint by tight junctions (figure 1A). Thus when the epithelial barrier is intact there is no escape of fluorescein in the lumen (figure 2A). However at sites of cell shedding fluorescein can sometimes be seen to enter the gap in the epithelium left by the shedding cell (figure 2B). Remarkably at sites of localised loss of barrier function in the epithelium we observed plumes of fluorescein efflux through epithelial layer at sites of epithelial cell loss. Such sites of loss of localised barrier function were confined to between two cells, as fluorescein loss was not seen from between nearby epithelial cells (figure 2C). Confocal endomicroscopy was also able to identify microerosions, where more than one cell is lost from a single site and the lamina propria is exposed to the lumen (figure $2 \mathrm{E}$ ). However, the microerosions were only visible with endomicroscopy, the white light imaging showed no visible epithelial damage or inflammatory change.

\section{The relationship between cell shedding and fluorescein efflux}

In general it is not possible to follow cellular events with CLE for more than a few seconds. We therefore performed in vivo confocal microscopy of cell shedding in mouse small intestine to define the relationship between cell shedding and fluorescein efflux more precisely. ${ }^{16} 26$ Alexa-fluor 647 conjugated dextrans were administered to the mouse intravenously as an analogue of the fluorescein used in our human studies. We found that instances of fluorescein efflux into the lumen from single sites on the mucosal surface were preceded by cell shedding at that site (figure 3A, online supplementary movie).

\section{Direction of flow at sites of loss of local barrier function}

There is currently great interest in the hypothesis that loss of intestinal barrier function enables entry of antigens and other toxic molecules from the intestinal lumen into intestinal mucosa, thereby triggering intestinal disease. ${ }^{30}$ We therefore analysed whether the direction of flow at the sites of loss of local barrier function was always outwards from the intestinal wall into the lumen as suggested by the observations above using fluoresceinaided endomicroscopy and the experience from our human study. Alexa-fluor 647 conjugated dextran was administered intravenously in murine in vivo preparations in order to visualise the villus vasculature and potential efflux from the intestine into the 
Figure 1 Confocal endomicroscopic imaging of epithelial cell shedding in the terminal ileum. (A) Fluorescein images capillaries beneath epithelial cells (block arrow) and the lateral intracellular space between epithelial cells (line arrow). (B) Epithelial cells become permeable to fluorescein prior to shedding (line arrow). (C-E) Fluorescein fluorescence signal is intense as shedding cells move out of the epithelial monolayer. (F) Intensely fluorescent shedding epithelial cells seen en face.
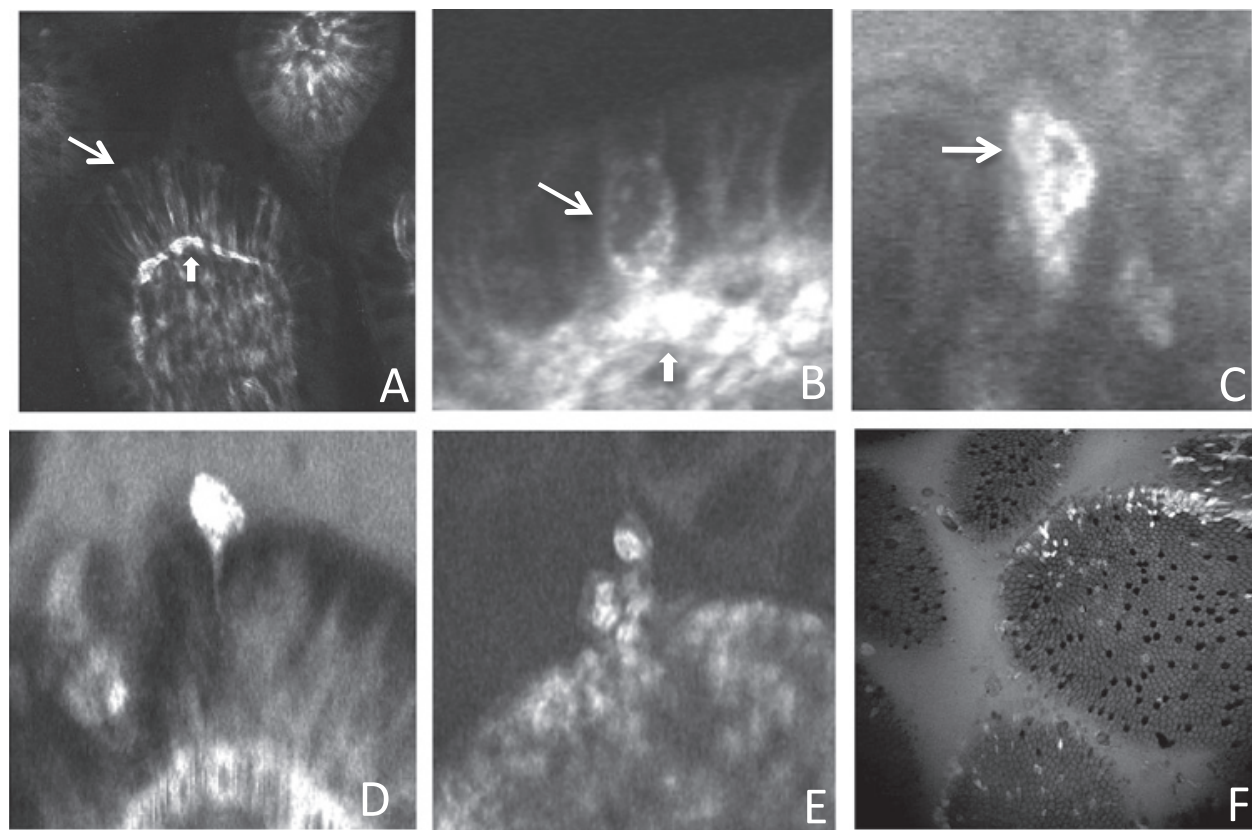

lumen. At the same time FITC-conjugated dextrans were applied to the luminal surface in a solution of $300 \mathrm{mOsm} / 1$ in order to assess influx across the intestinal barrier. Fields of view were analysed for cell shedding by the movement of Hoechst 33342 stained nuclei out of a villus and into the luminal compartment. We observed four patterns of flow at the gaps created by shedding cells (figure 3B-E). Examples of outward flow could be observed in which there was a continuous flow of intravenous dye (red) from the villus vasculature but there was no entry of the luminal dye (green) (figure 3B). Conversely we could observe instances in which there was entry of the luminal dye across the epithelial layer to the capillary arcade without efflux of the intravenous dye (figure $3 \mathrm{C}$ ). We also observed instances where there was simul- taneous influx and efflux of dyes (figure 3D) and instances where although there was an epithelial gap there was neither influx nor efflux of dyes (figure 3E).

The proportion of cell shedding events when the small intestinal epithelium was bathed in $300 \mathrm{mOsm} / 1$ in which there was leakage of dye in either direction was $18.8 \pm 0.9 \%$ (figure $4 \mathrm{~A}), 17.5 \pm 6.0 \%$ of which was inward, $76.3 \% \pm 10.3 \%$ was outward and $6 \pm 6.3 \%$ was in both directions (figure $4 \mathrm{~B}$ ). It should be noted that cell shedding events were only quantified when the origin and destination of the shed cell could be clearly observed within a z-stack of images over time.

We then asked if the direction of flow was influenced by the osmolarity of the luminal contents. Reduction of luminal
Figure 2 Loss of barrier function visualised by confocal endomicroscopy. (A) Intact barrier function with no escape of fluorescein into the gut lumen (arrow). (B) Fluorescein in the gap in the epithelium left by a shedding cell (arrow). Cellular debris from the shedding cell can be seen in the lumen. (C) efflux of fluorescein out of blood vessels (block arrow) into the lateral intercellular space. Efflux into the lumen is constrained at the apical border (block arrows). A plume of fluorescein effluxing through the gap left behind a shedding cell (line arrow). (D) Multiple sites of efflux of fluorescein through the epithelium into gut lumen (arrows). Note the increased fluorescence in the gut lumen. $(E)$ Microerosion (arrow) where more than one epithelial cell has been lost at one site exposing a capillary to the lumen. Note the functional relevance of this lesion as there is efflux of fluorescein into the lumen.
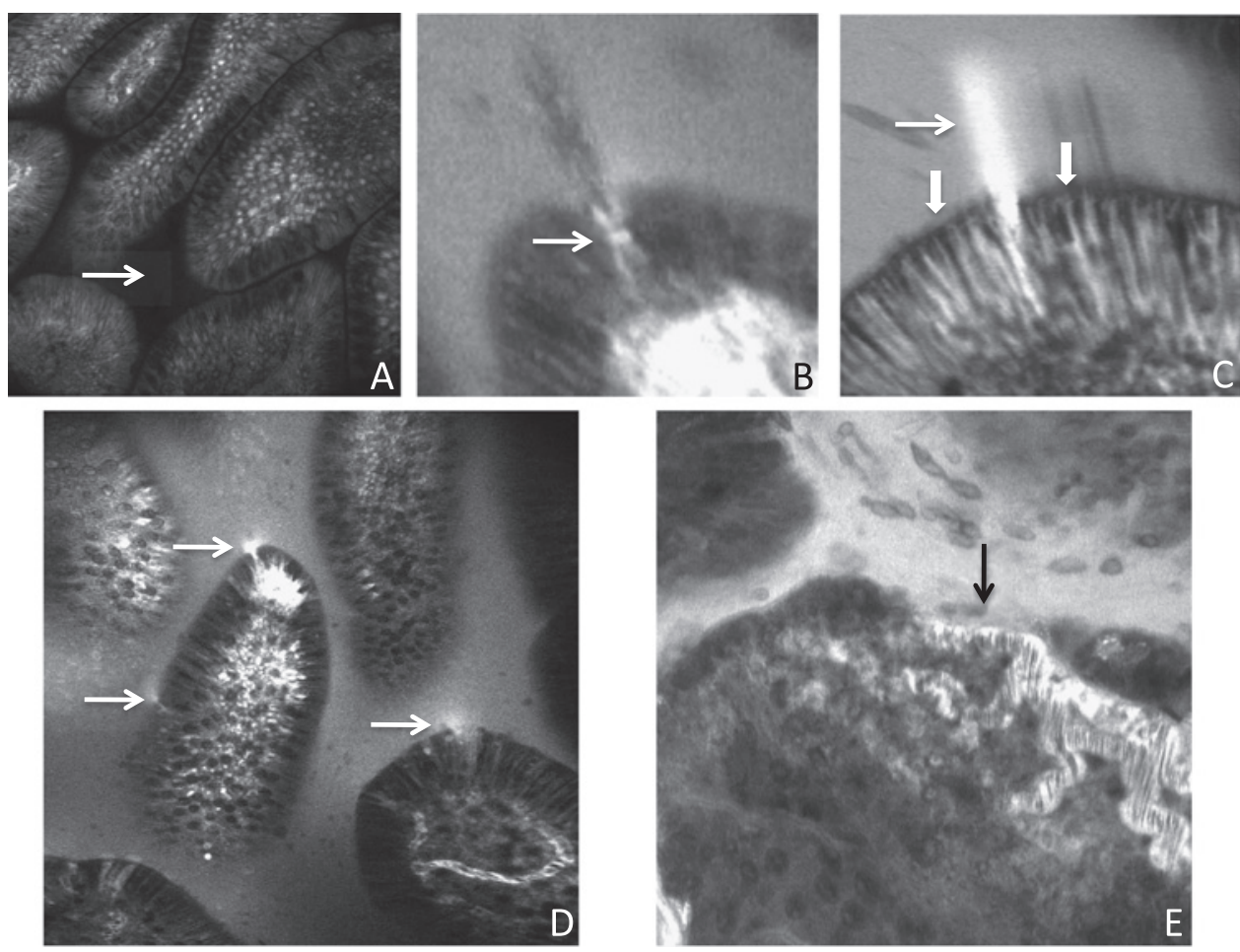

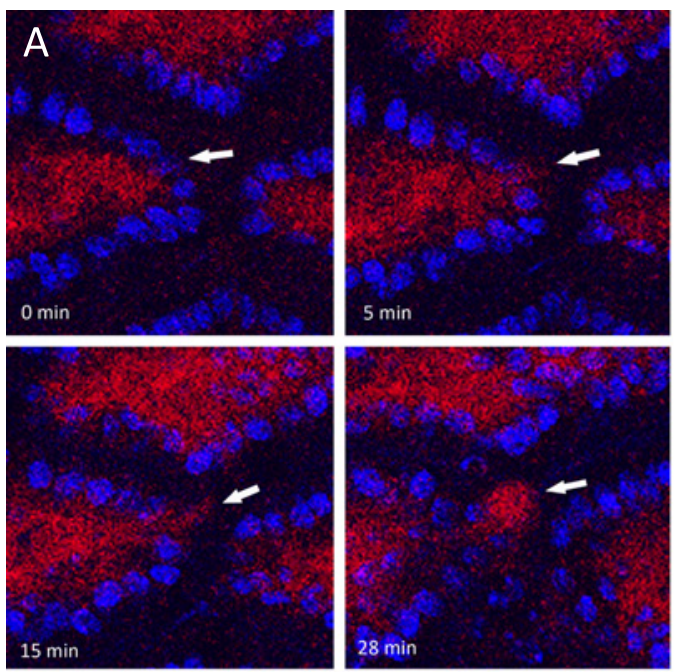
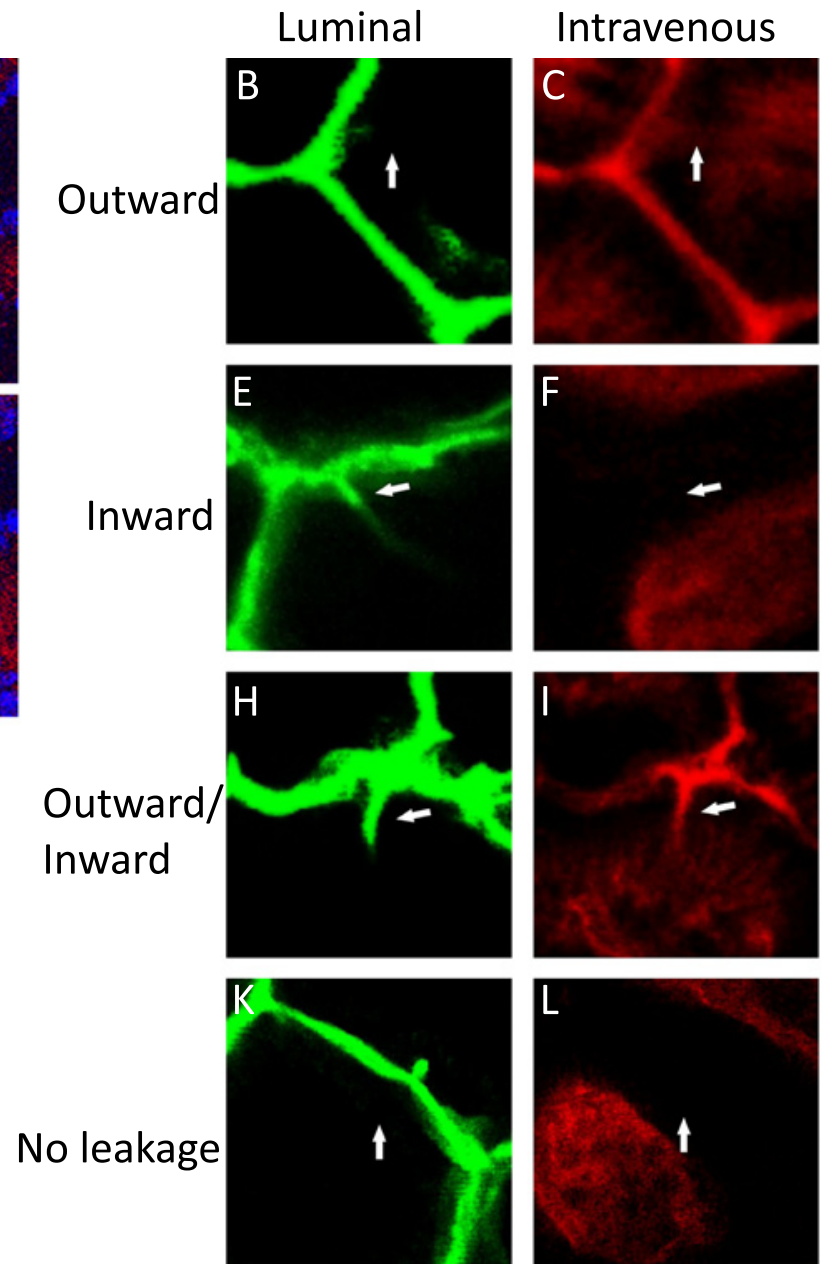
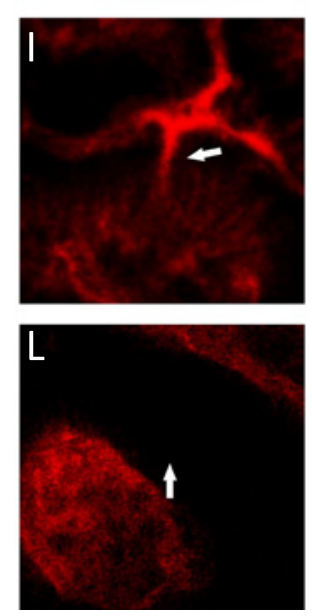

Combined
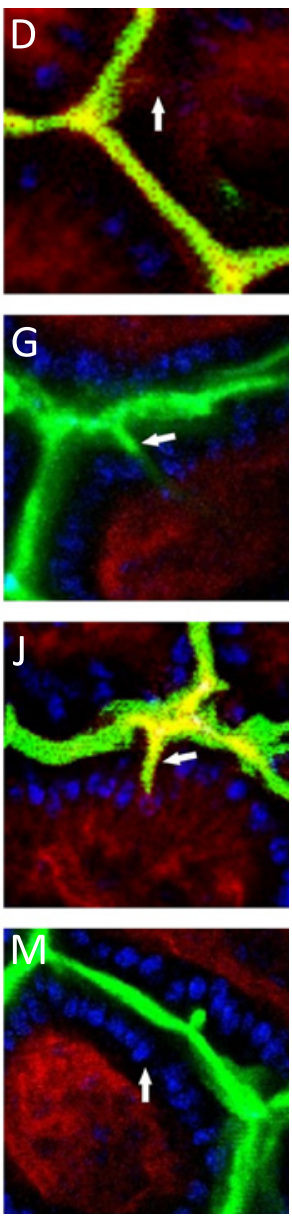

Figure 3 (A) Localised fluorescein leak is preceded by cell shedding. Intravenous Alexa-dextran 647 (MW 10000) shown in red and Hoechst 33342 labelled nuclei in blue. Images at 0, 5, 15 and 28 min relative to the start of cell shedding. (B-E) Dextran movement across small intestinal epithelia. Luminal FITC-dextran (MW 4000) shown in green. Intravenous Alexa-dextran 647 (MW 10000) shown in red and combined images shown with Hoechst 33342 labelled nuclei. Dextran leakage from circulation into lumen in an outward direction (B-D), from lumen in an inward direction ( $E-G)$, in both inward and outward directions $(\mathrm{H}-\mathrm{J})$ and no movement of inward or outward dextran $(\mathrm{K}-\mathrm{M})$.

osmolarity to $246 \mathrm{mOsm} / \mathrm{l}$ increased the abundance of leakageassociated cell shedding $(p<0.05)$ (figure $4 \mathrm{~A})$. Reduction in luminal osmolarity relative to plasma would be predicted to increase the driving force favouring inward flow. Appropriately we observed a substantial reduction in the proportion with outward flow $(p<0.05)$ a non-significant increase in the proportion of shedding events with inward flow, and a significant increase in the proportion with both inward and outward flow $(p<0.01)$ (figure $4 B)$.

\section{Incidence of gaps, fluorescein leakage and microerosions in patients with IBD}

We evaluated in a retrospective study the incidence of epithelial gaps, showed significantly more epithelial gaps, microerosions and fluorescein leakage in patients with UC and CD compared with controls (online supplemental table 1). We have shown previously that cell shedding causes discontinuities in the epithelium called 'gaps'. ${ }^{13} 14$ Images were only taken from areas of the colon or terminal ileum that were not inflamed or damaged on conventional colonoscopic views. There were significantly more epithelial gaps, microerosions and fluorescein leakage in patients with UC and CD than control patients (table 2) $(p<0.0001)$, indicating increased cell shedding and barrier loss in IBD patients. There was no significant difference between the endomicroscopic features of local barrier dysfunction in patients with CD and UC.

Prospective pilot study of the predictive value of cell shedding, gap formation and barrier loss for relapse of IBD

In order to quantify cell shedding and local barrier dysfunction at CLE we developed a grade based on the human and animal experiments described above and validated the grade by its prediction of subsequent relapse of $\mathrm{CD}$ and $\mathrm{UC}$ (table 1). A Watson grade of I is normal, physiological cell shedding defined as single cells being shed. In preliminary versions of the grade we found that the number of cells being shed did not predict subsequent relapse, providing there was no fluorescein leakage or microerosions created by multiple cells being shed from a single site. Thus the number of shedding cells being shed is not part of Watson grade I. Evidence of loss of barrier function when fluorescein leaks into the lumen is designated Watson grade II (functional defect). Microerosions caused by multiple epithelial cells being shed from a single site revealing the lamina propria to the lumen is designated Watson grade III (structural defect).

To validate the Watson grade we performed a prospective pilot study. Fifty-eight patients with IBD in complete clinical 
Figure 4 Analysis of loss of local barrier function and prediction of relapse of inflammatory bowel disease (IBD). Dextran movement (Luminal FITC-dextran (MW 4000) and intravenous Alexa-dextran 647 (MW $10000))$ through small intestinal epithelium at the site of epithelial cell shedding from luminal perfusion solutions of either $300 \mathrm{m0sm} / \mathrm{l}$ (black) and $246 \mathrm{m0sm} / \mathrm{l}$ (grey) solutions. (A) Percentage of events that show no dextran movement (sealed) and dextran leakage (leakage) in either the inward or the outward directions. (B) Percentage movement of dextran in an inward, outward or inward and outward direction from the leakage group. ${ }^{*} p<0.05,{ }^{* *} p<0.01$, error bars show SEM ( $n=4$ mice per group). (C) Kaplan-Meier plot of relapse of IBD patients over 12 months after confocal laser endomicroscopy stratified according to their Watson grade.
A

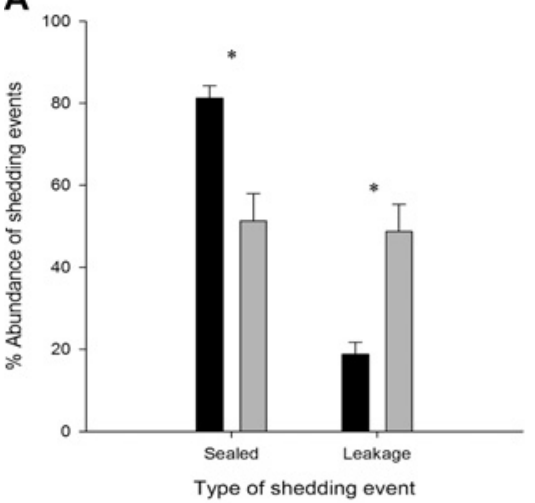

B

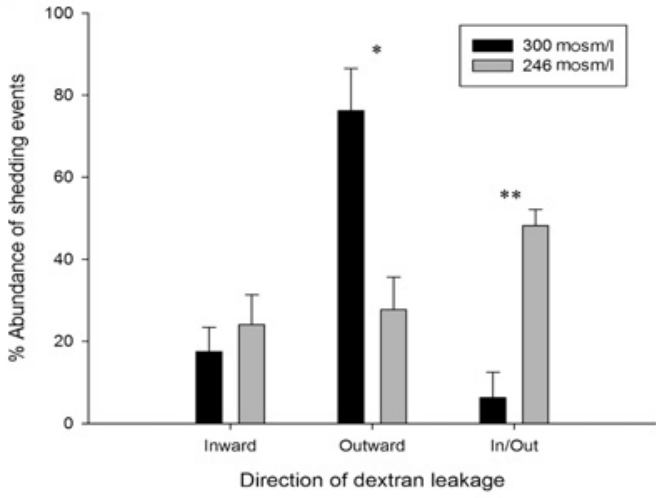

C

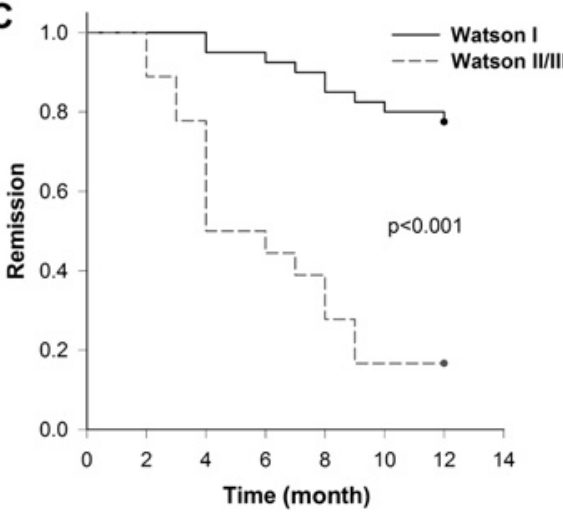

remission with absence of inflammatory changes on white light endoscopy were examined by confocal endomicroscopy. No serious or fluorescein or device related adverse events occurred during the prospective study. The endomicroscopic image grade was determined after the procedure. A total of 232 endomicroscopic images (four per patient) were judged. Cohen's $\mathrm{K}$ coefficient was 0.87 , which shows a strong agreement between the two blinded observers.

We found that 24 patients suffered a flare of their disease within 12 months after the confocal endomicroscopic examination. Kaplan-Meier analysis was undertaken of the relapse for the 12-month period after CLE following stratification by their Watson grade. Patients who subsequently had a flare of their disease had significantly more fluorescein leakage and microerosions thus a higher Watson grade than patients who did not suffer a flare $p<0.001$ (table 3, figure 4C). The sensitivity, specificity and accuracy for the Watson grades II/III to predict a flare were $62.5 \%$ (95\% CI 40.8 to 80.4$), 91.2 \%$ (95\% CI 75.2 to 97.7 ) and $79 \%$ (95\% CI 57.7 to 95.5$)$, respectively.

\section{DISCUSSION}

In this study we show that fluorescein-aided CLE can identify cells in the process of being shed. Remarkably, efflux of fluorescein can be seen at a proportion of sites of cell shedding providing for the first time direct visualisation of local barrier defects in human patients. Studies in mice demonstrate that both an efflux of intravenous dyes and an influx of luminal dyes can occur at sites of cell shedding. Furthermore, studies in mice show that the proportion of sites with influx or efflux is influenced by the osmotic gradient across the epithelium. Finally, we show that local defects in barrier function are increased and predict relapse in patients with IBD.

The morphology of the shedding cells in humans as demonstrated by CLE is remarkably similar to that observed in mice and is compatible with apoptosis. ${ }^{16} 2631$ Using intravenous administration of fluorescein we assessed the integrity of the epithelial barrier with CLE. In healthy individuals fluorescein does not enter the lumen though fluorescein can be seen tracking up the lateral intercellular space to the tight junctions (figure 1A, $\mathrm{B}$ and figure $2 \mathrm{~A}-\mathrm{C}$ ).

To our knowledge CLE is the only method that can identify sites of barrier loss at a microscopic level in real time. Although there are a number of physiological techniques to identify localised loss of barrier function they are not applicable to routine clinical usage. ${ }^{182-25} 3233$ The fact that CLE is combined with routine conventional colonoscopy makes it an attractive and convenient diagnostic methodology for routine clinical use. Flow of fluorescein from the bowel wall into the lumen is easy to detect as the luminal flow becomes fluorescent even if the site of barrier loss is not identified.

A recurrent theme in the current consensus view of the pathogenesis of IBD is that inward flow of antigens and

Table 2 Endomicroscopic features of local barrier dysfunction in IBD and controls

\begin{tabular}{|c|c|c|c|c|}
\hline & Crohn's disease & Ulcerative colitis & Controls & p Value* \\
\hline Patients (N) & 7 & 6 & 12 & NA \\
\hline $\begin{array}{l}\text { Average number of } \\
\text { optical biopsies }\end{array}$ & 33.8 & 42.1 & 36.6 & NS \\
\hline Gaps (\%) & $8.6 \pm 1.3$ & $8.2 \pm 2.1$ & $2.5 \pm 0.9$ & $<0.0001$ \\
\hline Microerosions (\%) & $1.8 \pm 0.6$ & $1.4 \pm 0.7$ & $0.1 \pm 0.03$ & $<0.0001$ \\
\hline $\begin{array}{l}\text { Fluorescein leakage } \\
\text { (\% of optical biopsies) }\end{array}$ & $32.4 \pm 9.3$ & $28.9 \pm 7.2$ & $4.2 \pm 3.1$ & $<0.0001$ \\
\hline
\end{tabular}

$\%$ Gaps and microerosions refer the proportion of the perimeter of epithelial surface with gaps or microerosions.

*p Value: $t$ test IBD patients (Crohn's disease and ulcerative colitis) versus controls. IBD, inflammatory bowel disease; NA, not applicable; NS, not significant. 
Table 3 Watson grade of cell shedding, fluorescein leakage and microerosions and prediction of flare over a 12-month period after confocal laser endomicroscopy

\begin{tabular}{lll}
\hline & No flare & Flare \\
\hline Watson grade & & \\
I & 31 & 9 \\
II & 3 & 8 \\
III & 0 & 7 \\
& & $\mathrm{p}<0.001$ (I vs II/III) \\
\hline
\end{tabular}

Data on the relationship between relapse of patients with inflammatory bowel disease over a 12-month period after determination of the Watson grade at confocal laser endomicroscopy (58 inflammatory bowel disease patients; 47 with ulcerative colitis and 11 with Crohn's disease).

microbes from the intestinal lumen into the bowel wall is of importance. ${ }^{4} 3435$ Our CLE studies in humans are only able to demonstrate flow in the opposite direction; in other words from within the bowel wall into the lumen. However we hypothesised that if there are sites of outward flow there might also be sites of inward flow, which would be more pathologically relevant, and that outward flow detected at CLE might be a marker of inward flow. We employed our mouse model of cell shedding to investigate further the local barrier defects at sites of cell shedding. ${ }^{26}$ Using intravenous Alexa-dextran 647 we showed that the localised barrier defects that we observed during human CLE could be the result of cell shedding (figure 3A). Furthermore we demonstrate that incomplete sealing of the site of cell shedding can result in outward flow, inward flow or bidirectional flow and that the osmotic gradients between the lumen and the epithelial monolayer are a factor that determines the direction of flow through the barrier defect (figures 3 and 4). The other factors that may determine the direction of flow through local barrier defects are hydrostatic pressure with the intestinal wall, villus contraction and peristalsis. ${ }^{36-38}$

We have demonstrated that while majority of shedding sites are sealed when the luminal contents are iso-osmolar with serum, the proportion of sealed sites is reduced by an inwardly directed osmotic flow (figure 4A). We also show that the direction of flow through epithelial barrier defects is influenced by luminal osmolarity. Imposing an inwardly directed osmotic gradient reduces outward flow and increases bidirectional flow, which we interpret as free diffusion back and forth (figure 4B). From these observations we conclude that the direction of flow through the epithelium is unstable and highly dependent on local osmotic and pressure gradients. While the luminal contents of the intestine are classically regarded as iso-osmolar, there are likely to be areas, particularly in the upper small intestine, where mixing and transepithelial ion transport has not achieved isoosmolarity within the lumen. Our finding that loss of barrier function predicts relapse (table 3, figure 4C) supports our conclusion that outward flow of fluorescein into the intestinal lumen identified by CLE is a marker of loss of barrier function where inward flow of antigens, toxins and microbes, activating the mucosal immune system also occurs.

CLE identifies three related parameters, cell shedding, flow of fluorescein into the intestinal lumen and the development of microerosions, which we combined into a simple grading system to predict relapse of with IBD in remission. In early versions of our grading system we were surprised that the abundance of cell shedding alone did not predict relapse. Only cell shedding with the addition of barrier loss was predictive. This is in accord with our earlier observations ${ }^{13} 1426$ that the complete sealing of gaps is achieved if inflammatory cytokine concentrations are low (figure 4A). The presence of microerosions (loss of more than one adjacent epithelial cell from a single site) was also predictive of relapse. Microerosions without loss of barrier function were uncommon so the presence of microerosions is likely to be a marker of loss of barrier function.

In a prospective study we examined 58 patients with IBD and complete mucosal healing as defined by white light endoscopy. The presence of functional (fluorescein leakage) or structural defects (microerosions) could be readily identified in the terminal ileum of patients using fluorescein-aided endomicroscopy and predicted relapse of IBD. Patients with local barrier dysfunction (Watson II/III) were significantly more likely to relapse $(p<0.001)$ compared with patients where healing was also confirmed endomicroscopically (Watson grade I). It is of interest that we found changes in the terminal ileum of patients with UC that predict relapse even though classically UC does not include the terminal ileum. We are investigating this finding further with additional studies. The data from this pilot study provides data to enable the design of a clinical trial to confirm whether CLE can accurately predict the relapse of patients with $\mathrm{UC}$ or $\mathrm{CD}$

In conclusion, these studies suggest CLE might be a useful tool for the management of IBD. The prediction and prevention of relapse of IBD is a major goal of current IBD clinical research. ${ }^{39}$ Our observations suggest that CLE might be a useful and convenient tool to predict relapse and adds to the growing body of evidence that loss of barrier function plays a major role in the pathogenesis of IBD. ${ }^{11} 1240$

Funding Wellcome Trust grant WT087768MA to AJMW and a grant from Association François Aupetit (AFA) to DM.

Competing interests RK has an unrestricted grant from Pentax Europe and has received instruments for free via Optiscan. All other authors have no competing interests.

Ethics approval The study was approved by the local ethical committee in Rheinland-Pfalz, Germany (No. 837.364.07).

Contributors RK, CAD, DM, LGL, MG, DMP, MFN and AJMW contributed to the study concept, design (except DMP) and were involved in analysis, interpretation of data and drafting of the manuscript. RK, CAD, DM, AG, LGL, MG and AJMW were involved in acquisition of data. RK, PRG, DMP, MFN and AJMW contributed to critica revision of the manuscript for important intellectual content. RK, DM, LGL and MG carried out the statistical analysis. RK, DMP, MFN and AJMW obtained funding. RK, MFN and AJMW were involved in study supervision.

Provenance and peer review Not commissioned; externally peer reviewed.

\section{REFERENCES}

1. Duerksen DR, Wilhelm-Boyles C, Parry DM. Intestinal permeability in long-term follow-up of patients with celiac disease on a gluten-free diet. Dig Dis Sci 2005:50:785-90.

2. Berkes J, Viswanathan VK, Savkovic SD, et al. Intestinal epithelial responses to enteric pathogens: effects on the tight junction barrier, ion transport, and inflammation. Gut 2003;52:439-51.

3. Meddings J. The significance of the gut barrier in disease. Gut 2008;57 438-40.

4. Marchiando AM, Graham WV, Turner JR. Epithelial barriers in homeostasis and disease. Annu Rev Pathol 2010;5:119-44.

5. Peeters M, Ghoos $Y$, Maes B, et al. Increased permeability of macroscopically normal small bowel in Crohn's disease. Dig Dis Sci 1994;39:2170-6.

6. Peeters M, Geypens B, Claus D, et al. Clustering of increased small intestinal permeability in families with Crohn's disease. Gastroenterology 1997;113 802-7.

7. May GR, Sutherland LR, Meddings JB. Is small intestinal permeability really increased in relatives of patients with Crohn's disease? Gastroenterology 1993; 104:1627-32.

8. Wyatt $\mathbf{J}$, Vogelsang $\mathrm{H}$, Hubl W, et al. Intestinal permeability and the prediction of relapse in Crohn's disease. Lancet 1993;341:1437-9.

9. Zeissig S, Burgel N, Gunzel D, et al. Changes in expression and distribution of claudin 2, 5 and 8 lead to discontinuous tight junctions and barrier dysfunction in active Crohn's disease. Gut 2007; 56:61-72.

10. Marchiando AM, Shen L, Graham WV, et al. Caveolin-1-dependent occludin endocytosis is required for TNF-induced tight junction regulation in vivo. J Cell Biol 2010;189:111-26. 
11. Su L, Shen L, Clayburgh DR, et al. Targeted epithelial tight junction dysfunction causes immune activation and contributes to development of experimental colitis. Gastroenterology 2009;136:551-63.

12. Arrieta MC, Madsen K, Doyle J, et al. Reducing small intestinal permeability attenuates colitis in the IL10 gene-deficient mouse. Gut 2009;58:41-8.

13. Guan Y, Watson AJ, Marchiando AM, et al. Redistribution of the tight junction protein ZO-1 during physiologic shedding of mouse intestinal epithelial cells. Am J Physiol Cell Physiol 2011;300:C1404-14.

14. Kiesslich R, Goetz M, Angus EM, et al. Identification of epithelial gaps in human small and large intestine by confocal endomicroscopy. Gastroenterology 2007;133:1769-78.

15. Watson AJ, Duckworth CA, Guan Y, et al. Mechanisms of epithelial cell shedding in the Mammalian intestine and maintenance of barrier function. Ann N Y Acad Sci 2009;1165:135-42.

16. Marchiando AM, Shen L, Graham VW, et al. The epithelial barrier is maintained by in vivo tight junction expansion during pathologic intestinal epithelial shedding. Gastroenterology 2011:140:1208-18.

17. Liu JJ, Wong K, Thiesen AL, et al. Increased epithelial gaps in the small intestines of patients with inflammatory bowel disease: density matters. Gastrointest Endosc 2011:73:1174-80.

18. Arrieta MC, Bistritz L, Meddings JB. Alterations in intestinal permeability. Gut 2006:55:1512-20.

19. Meddings JB, Sutherland LR, Byles NI, et al. Sucrose: a novel permeability marker for gastroduodenal disease. Gastroenterology 1993;104:1619-26.

20. Hilsden RJ, Meddings JB, Sutherland LR. Intestinal permeability changes in response to acetylsalicylic acid in relatives of patients with Crohn's disease. Gastroenterology 1996;110:1395-403.

21. Munkholm P, Langholz E, Hollander D, et al. Intestinal permeability in patients with Crohn's disease and ulcerative colitis and their first degree relatives. Gut 1994;35:68-72.

22. Schulzke JD, Fromm M, Bentzel CJ, et al. Adaptation of the jejunal mucosa in the experimental blind loop syndrome: changes in paracellular conductance and tight junction structure. Gut 1987;28:159-64.

23. Kroesen AJ, Dullat S, Schulzke JD, et al. Permanently increased mucosal permeability in patients with backwash ileitis after ileoanal pouch for ulcerative colitis. Scand J Gastroenterol 2008;43:704-11.

24. Fromm M, Krug SM, Zeissig S, et al. High-resolution analysis of barrier function. Ann N Y Acad Sci 2009;1165:74-81.
25. Gitter AH, Wullstein F, Fromm M, et al. Epithelial barrier defects in ulcerative colitis: characterization and quantification by electrophysiological imaging. Gastroenterology 2001;121:1320-8.

26. Watson AJ, Chu S, Sieck $L$, et al. Epithelial barrier function in vivo is sustained despite gaps in epithelial layers. Gastroenterology 2005;129:902-12.

27. Gunzel D, Florian P, Richter JF, et al. Restitution of single-cell defects in the mouse colon epithelium differs from that of cultured cells. Am J Physiol Regul Integr Comp Physiol 2006;290:R1496-507.

28. Vermeire $\mathbf{S}$, Schreiber $\mathbf{S}$, Sandborn WJ, et al. Correlation between the Crohn's disease activity and Harvey-Bradshaw indices in assessing Crohn's disease severity. Clin Gastroenterol Hepatol 2010;8:357-63.

29. Hirai F, Matsui T, Aoyagi $\mathrm{K}$, et al. Validity of activity indices in ulcerative colitis: comparison of clinical and endoscopic indices. Dig Endosc 2010;22:39-44.

30. Shen L, Weber CR, Raleigh DR, et al. Tight junction pore and leak pathways: a dynamic duo. Annu Rev Physiol 2011:73:283-309.

31. Bullen TF, Forrest $S$, Campbell $F$, et al. Characterization of epithelial cell shedding from human small intestine. Lab Invest 2006;86:1052-63.

32. Gitter AH, Bertog M, Schulzke J, et al. Measurement of paracellular epithelial conductivity by conductance scanning. Pflugers Arch 1997;434:830-40.

33. Epple HJ, Schneider T, Troeger H, et al. Impairment of the intestinal barrier is evident in untreated but absent in suppressively treated HIV-infected patients. Gut 2009:58:220-7.

34. Salim SY, Soderholm JD. Importance of disrupted intestinal barrier in inflammatory bowel diseases. Inflamm Bowel Dis 2011;17:362-81.

35. Fritz T, Niederreiter L, Adolph T, et al. Crohn's disease: NOD2, autophagy and ER stress converge. Gut 2011;60:1580-8.

36. Lee JS. Contraction of villi and fluid transport in dog jejunal mucosa in vitro. Am $\mathrm{J}$ Physiol 1971;221:488-95.

37. Lee JS. Epithelial cell extrusion during fluid transport in canine small intestine. Am J Physiol 1977;232:E408-14.

38. Moore R, Carlson S, Madara JL. Villus contraction aids repair of intestinal epithelium after injury. Am J Physiol 1989;257:G274-83.

39. Ahmed T, Rieder F, Fiocchi $C$, et al. Pathogenesis of postoperative recurrence in Crohn's disease. Gut 2011:60:553-62.

40. Su L, Nalle SC, Sullivan EA, et al. Genetic ablation of myosin light chain kinase limits epithelial barrier dysfunction and attenuates experimental inflammatory bowel disease. Gastroenterology 2009;136:abstract. 